\title{
Segmentação de imagens de ultrassonografia da carcaça em pequenos ruminantes utilizando Deep Learning
}

\author{
Tiago M Oliveira ${ }^{1}$, José L R Sarmento ${ }^{2}$, Luiz A S Figueiredo Filho ${ }^{3}$, Romuere R V Silva ${ }^{1}$ \\ ${ }^{1}$ Sistemas de Informação, UFPI, Picos - PI - Brazil \\ ${ }^{2}$ Departamento de Zootecnia, UFPI, Teresina - PI - Brazil \\ ${ }^{3}$ Campus Caxias, Instituto Federal do Maranhão (IFMA), Caxias - MA - Brazil
}

romueredufpi.edu.br

\begin{abstract}
Brazil is one of the main countries operating in the agribusiness sector. Sheep and goat farming is one of the segments of Brazilian agribusiness. The evaluation of each carcass of goats and sheep is carried out by a specialist who evaluates them based on visual aspects, being susceptible to errors in the final evaluation. In this context, the purpose of this work is to use Convolutional Neural Networks to segment the Longissimus dorsi muscle area in ultrasonographic images of small ruminants. Our experiments showed that the PSPNet $C N N$ architecture achieved the results with an Intersect over Union (IoU) rate of 0.89. It was possible to obtain a precise segmentation of the images, which will allow the producer to correctly diagnose the measurements of the animals with greater practicality and saving time.
\end{abstract}

\section{Introdução}

A ovinocaprinocultura, refere-se à criação de ovinos e caprinos com a finalidade de produção de carne, lã, couro, leite e outros derivados. No Brasil é uma atividade que encontra-se em constante crescimento. $\mathrm{O}$ atual quadro consta 18.948 .934 ovinos e 10.696.664 caprinos, destacando a parte nordeste do país que detém 93,93\% e 66,67\% dos rebanhos, respectivamente [IBGE 2020].

No Brasil esta atividade demonstra um importante papel no contexto do agronegócio, especialmente por conta da geração de oportunidades de emprego, renda e fixação do homem no campo. No entanto, é ainda precário em alguns pontos como no nível de produtividade, de gerenciamento, e de articulação entre os diversos elos da cadeia produtiva da carne e pele, dificultando a sustentabilidade, a competitividade e a remuneração dos produtores [ROSANOVA 2004].

A atividade de classificação das carcaças em ovinos e caprinos tem um impacto significativo no preço final dos produtos gerados pela indústria de produção animal. A classificação do preço e da qualidade da carne que será fornecida ao consumidor final depende de uma classificação realizada por um especialista que consiste em uma atividade longa, cansativa e trabalhosa que avalia a qualidade com base em uma inspeção visual de suas características externas. Com base nessas características e nos critérios padronizados, o especialista atribui à carcaça do animal uma classificação e nível de gordura específicos.

Utilizar tecnologias emergentes já consolidadas que foram aplicadas há outras espécies torna-se algo primordial, como empregado na avaliação de carcaça de imagens 
ultrassonográficas, é importante inserir tais técnicas na criação de ovinos e caprinos para contribuir no atendimento do mercado consumidor em crescimento [Neto 2010]. Segundo [Tauroco 2020], o método de avaliação da carcaça em tempo real por ultrassonografia caracteriza-se por ser não invasivo e não deixa resíduos nocivos na carne, oferecendo meios objetivos de avaliar os animais vivos em relação a sua composição corporal.

Uma das principais regiões que podem ser analisadas e que são importantes para o mensuração da qualidade de carcaça é o músculo Longissimus dorsi [Cartaxo and Sousa 2008]. Nessa região é possível mensurar diferentes características, as mais importantes são: a área de olho de lombo (AOL), utilizada como característica indicadora de musculosidade e de qualidade; e, a espessura de gordura subcutânea (EGS), que indica o grau de acabamento da carcaça e se relaciona com a qualidade da carne por proteger a carcaça durante o processo de resfriamento.

Métodos tradicionais dependem de um especialista da área (médico veterinário ou zootecnista) para segmentar manualmente a região do músculo Longissimus dorsi na imagem de ultrassom, e a partir disso as características de AOL e EGS podem ser mensuradas. Nesse contexto, o propósito deste trabalho é utilizar técnicas de visão computacional para segmentar a área do músculo Longissimus dorsi em imagens ultrassonográficas de pequenos ruminantes.

O restante deste artigo está organizado da seguinte forma. A Seção 2 mostra os trabalhos relacionados. A Seção 3 descreve a metodologia proposta. A Seção 4 descreve os resultados experimentais e as discussões com explicações. Finalmente, a Seção 5 apresenta as conclusões.

\section{Trabalhos Relacionados}

Esta seção apresenta um levantamento do estado da arte dos métodos existentes para a avaliação de qualidade da carcaça de ruminantes em imagens de ultrassom, utilizando técnicas de visão computacional em imagens.

No trabalho de [Font-i Furnols et al. 2014], foi feito previsões da composição e da gordura intramuscular (IMF) de seções de costela de boi que foram estimadas a partir de 1 imagem transversal tirada no nível da $10^{\mathrm{a}}$ costela ao nível da $11^{\mathrm{a}}$ costela IMF ou de uma série de imagens transversais tiradas entre a $9^{\mathrm{a}}$ e $11^{\mathrm{a}}$ costelas com scanner de tomografia computadorizada (TC). As amostras de costelas foram escaneadas com equipamento de scanner CT HiSpeed Zx/i CT da General Electric na IRTA-CENTA em Monells (Girona, Espanha). Três varreduras foram feitas de cada seção de costela. Os dados foram divididos aleatoriamente em um conjunto de calibração (2/3 dos dados) e um conjunto de validação (1/3 dos dados). Os dados das imagens de calibração foram analisados por regressão linear. $\mathrm{O}$ trabalho mostra diferentes maneiras de estimar o conteúdo do IMF. A partir de uma imagem em corte transversal de $10 \mathrm{~cm}$ de espessura tirada no nível da $10^{a}$ costela. O IMF também foi previsto a partir do histograma da Região de interesse do músculo Longissimus, que se relaciona mais de perto com a posição da qual a amostra foi retirada para a medição de referência do IMF. Nesse caso, a precisão diminuiu significativamente e não foi bem validada com o conjunto de dados externo. Uma combinação de distribuições de volume de acordo com os valores de toda a imagem transversal e da região de interesse do músculo $L$. thoracis resultou em graus semelhantes de precisão em contraste com o uso de uma única imagem. O conteúdo do IMF foi estimado a partir de 
todas as imagens da seção de costela e a precisão resultante não melhorou. Nesse caso, o conjunto de validação produziu um grau de precisão semelhante ao conjunto de resultados estatísticos.

[Peña et al. 2014], propôs um estudo com objetivo de avaliar a capacidade preditiva de imagens de ultrassom em diferentes idades com base na composição do corte da costela $10^{\mathrm{a}}-12^{\mathrm{a}}$. Aplicando múltiplos coeficientes de regressão em diferentes varreduras para a determinação de tecidos cortados da $10-12^{\mathrm{a}}$ costela de raças Charolês, Limousin e Retinta. As medições de ultrassom feitas em todas as raças forneceram baixa precisão de predição para a composição do corte $10-12^{\text {a }}$ costela $R^{2}=0,12$ e $R^{2}=0,46$. Por outro lado, foi mostrado evidências claras de que os melhores coeficientes de determinação para a predição das porcentagens de tecido cortado na $10^{\mathrm{a}}-12^{\mathrm{a}}$ costela estão em touros Retinta. Nessa raça, o resultado da regressão múltipla obtida na última varredura antes do abate foram responsáveis por $46 \%, 43 \%$ e $44 \%$ da variação na porcentagem de massa magra, gordura e osso, respectivamente. Os coeficientes de regressão melhoraram com a inclusão de duas ou três varreduras. Semelhante quando foi considerado um único escaneamento, a precisão das previsões aumenta com a diminuição do tempo entre o escaneamento e o abate. Em geral, esses coeficientes aumentaram ao considerar as medidas de ultrassom de vários exames que apenas um ponto de tempo foi usado.

[Oliver et al. 2010], propuseram uma análise por regressão múltipla que trata duas ou mais variáveis explicativas ao contrário da regressão linear que trata somente uma variável explicativa, a regressão múltipla do peso da carcaça e do escore de conformação do Sistema Europeu de Classificação de Carcaça (SEUROP) no rendimento de carne e os quatro rendimentos da categoria de qualidade de corte comercial (extra, primeiro, segundo e terceiro) foi realizada como uma medida da precisão do sistema SEUROP. Foi realizada a análise de regressão stepwise do peso da carcaça e as 33 medidas de análise morfométrica da imagem e rendimento de carne e rendimentos das quatro categorias de qualidade de corte comercial. A maior precisão foi alcançada usando análise de imagem. Os valores do coeficiente de regressão estavam entre $R^{2}=0,66$ e $R^{2}=0,93(\mathrm{P}<0,001)$ para o sistema SEUROP e entre $R^{2}=0,81$ e $R^{2}=0,94(\mathrm{P}<0,001)$ para o método de análise de imagem. Os resultados sugerem que o método de análise de imagem deve ser útil como um meio de complementar e melhorar o sistema SEUROP para classificação de carcaças de bovinos. O peso da carcaça foi a variável que melhor explicou a composição da carcaça. Se o peso da carcaça for restrito à primeira variável independente, a precisão adicional alcançada pela incorporação de medidas mais caras pode então ser avaliada [Kempster et al. 1982].

[Daniel et al. 2020], propuseram um projeto de um sistema de visão computacional em combinação com um sistema de sensoreação para a classificação em tempo real de carcaças de bovinos. Na etapa de Pré-processamento e seleção de contorno, foi corrigido e preparada cada uma das imagens para homogeneizar a amostra de dados selecionando o contorno (silhueta) da carcaça e extrair seus pixels, uma vez obtida a borda da imagem, o próximo passo na etapa de Detecção de pontos de referência para segmentação de carcaça bovina é dividir a carcaça em diferentes regiões. Esta divisão é formada por uma série de linhas imaginárias que os especialistas usam para classificar a carcaça. Para fazer esta divisão, a técnica de detecção de pontos de referência[Jain and Li 2011], comumente usado em processos de reconhecimento facial. Seguindo o trabalho realizado pelos auto- 
res em [Lindner et al. 2016], O Random Forest Regression-Voting [Cutler et al. 2012] é aplicado por ser um sistema rápido de treinar e avaliar. O processo de correção de cores é realizado na fase de pré-processamento da imagem. Este processo é essencial ao fazer uma estimativa correta e normalizada do nível de gordura presente na carne. Uma vez detectada a gordura na imagem completa da carcaça, o percentual de gordura em cada uma das divisões de corte é calculada. Para esta tarefa, a divisão feita através dos pontos de segmentação calculados são aplicados. Após a obtenção dos resultados de um conjunto de carcaças bovinas reais, foi possível concluir que o sistema pode prever, com alta precisão, os resultados da classificação feita por um conjunto de 6 especialistas. No tempo que um especialista humano levaria para classificar um total de 40 peças, o sistema pode classificar com precisão até 10 vezes mais peças e enviar os resultados em tempo real para o sistema.

No presente trabalho analisamos imagens da AOL de pequenos ruminantes, que utilizamos como característica indicadora de musculosidade e de qualidade da carne.

\section{Método Proposto}

Este trabalho propõe o desenvolvimento de um sistema computacional para avaliação de carcaça de pequenos ruminantes por meio de reconhecimento de imagens ultrassonográficas. Dessa forma, nossa metodologia proposta consiste em 3 etapas: 1) aquisição de um conjunto de imagens; 2) aplicação de técnicas de pré-processamento; 3) treinamento de uma Rede Neural Convolucional (CNN) para classificação de imagens.

\subsection{Aquisição do conjunto de imagens}

O banco de dados utilizado na pesquisa é composto por informações mensuradas em ovinos e caprinos, destinados a abatedouros, criados nos estados do Maranhão e Piauí, que compõem a região Meio-Norte do Brasil, em propriedades de criadores com animais registrados em associações de criadores. Participam da pesquisa animais de ambos sexos com idade adulta no momento da coleta de dados.

$\mathrm{Na}$ avaliação da carcaça in vivo, são realizadas captura de imagens ultrassonográficas por meio de aparelho de ultrassom equipado com transdutor linear de 13 $\mathrm{cm}$, utilizando-se ajuste da frequência de 5,0 MHz. Para avaliar a musculosidade são mensuradas as características: área de olho de lombo (AOL), profundidade de olho de lombo (POL) e comprimento de olho de lombo (COL), todas avaliadas através de imagens de ultrassom corte transversal do músculo Longissimus dorsi, tendo-se como referência anatômica o espaço intercostal entre a $12^{a}$ e $13^{a}$ costela. A AOL é dada em $\mathrm{cm}^{2}$, enquanto que POL e COL em cm. Além disso, o conjunto de imagens possui a marcação do músculo Longissimus dorsi.

Durante a captura das imagens ultrassonográficas, houve uma contenção do animal em estudo, de maneira confortável e relaxada para obtenção de uma maior qualidade das imagens. Também foi realizado a mensuração das características supracitadas por meio da ferramenta de medidas do próprio aparelho de ultrassom a fim de possibilitar a validação das avaliações posterior ao desenvolvimento do software de reconhecimento de imagens ultrassonográficas de características de carcaça, dessa forma foram obtidas 206 imagens. A Figura 1 mostra exemplos de imagens de nosso banco de dados bem como as suas respectivas marcações. 


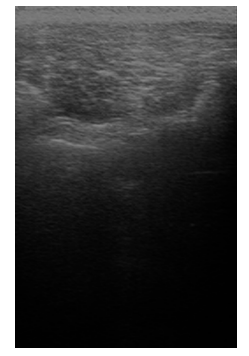

(a)

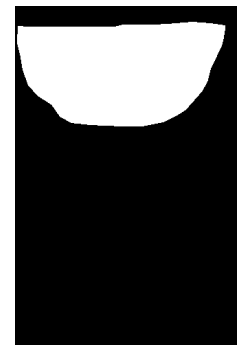

(b)

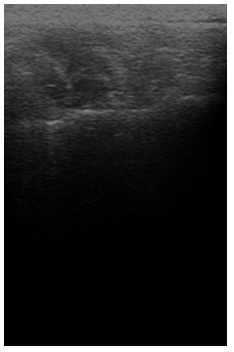

(c)

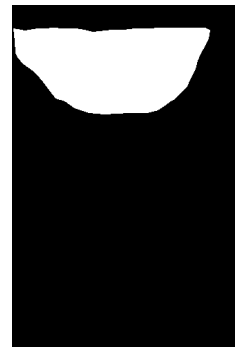

(d)

Figura 1. Exemplos de imagens do banco de dados. Em (a) e (c) estão imagens de ultrassonografia, e em (b) e (d) suas respectivas regiões do músculo Longissimus dorsi marcadas.

\subsection{Pré-processamento de Imagens}

Os modelos CNN usados têm como entrada imagens de tamanho quadrado $(320 \times 320)$ e (384 × 384), e é necessário redimensionar as imagens para essas dimensões. Além disso, os modelos CNN esperam valores de pixel entre 0 e 1 . Nossas imagens têm valores de pixel entre 0 e 255. Dessa forma, realizou-se um ajuste linear, escalar, transformação dos níveis de cinza dos pixels para se adequarem aos requisitos do $\mathrm{CNN}$.

\subsection{Treinamento do Modelo CNN para Segmentação de Imagens}

Neste trabalho, aplicamos quatro diferentes arquiteturas CNN pré-treinadas: U-Net proposta por [Ronneberger et al. 2015]; FPN proposto por [Lin et al. 2017]; LinkNet proposto por [Chaurasia and Culurciello 2017]; e PSPNet proposta por [Zhao et al. 2017]. A Tabela 1 mostra as características das arquiteturas $\mathrm{CNN}$ utilizadas neste trabalho. $\mathrm{O}$ treinamento desses modelos foi realizado por 100 épocas, foram definidos alguns parâmetros para realizar o treinamento do modelo com base nas arquiteturas CNNs pré-treinadas. Esses parâmetros são definidos na Tabela 1.

Tabela 1. Parâmetros usados para segmentação.

\begin{tabular}{l|l}
\hline Tipo & Parâmetros \\
\hline CNN & U-Net, FPN, LinkNet e PSPNet \\
Função de Ativação & Sigmoid \\
Otimizador & Adam \\
Função de Perda & Jaccard Distance \\
Batch Size & 16 \\
Épocas & 100 \\
Backbone & Resnet34 \\
Métricas & F1-Score e IOU Score \\
\hline
\end{tabular}

\section{Resultados}

Nesta seção, relatamos os resultados obtidos em nossos experimentos. Para calcular a qualidade da segmentação obtida pelas redes neurais utilizamos três métricas: loss, IOU (Intersect over Union) e F1-Score [Nowozin 2014]. Nossa metodologia de segmentação usou CNNs pré-treinadas combinados com pré-processamento. O conjunto de imagens 
Tabela 2. Resultados obtidos pela metodologia proposta para cada modelo.

\begin{tabular}{l|l|l|l}
\hline Modelo & Loss & IOU & F1-Score \\
\hline U-Net & 0,19 & 0,89 & 0,94 \\
FPN & 0,19 & 0,88 & 0,93 \\
LinkNet & 0,20 & 0,89 & 0,94 \\
PSPNet & 0,16 & 0,89 & 0,94 \\
\hline
\end{tabular}

foi divido em $60 \%$ para treinamento $20 \%$ para validação e $20 \%$ para teste. Os resultados obtidos são apresentados na Tabela 4.

Os resultados dos modelos foram semelhantes, porém o PSPNet se sobressai pelo fato de ter obtido o menor valor na função de loss com 0,16, IOU de 0,89 e F1-Score de 0,94. A Figura 2 demonstra o gráfico de treino e validação na métrica F1-Score nesse modelo, observa-se que a partir da época 40 existe pouca variação no aprendizado da rede. Isso mostra que a rede convergiu para um resultado robusto em uma quantidade de épocas menor quando comparado com outros trabalhos que usam esse tipo de CNN [Araújo et al. 2021].

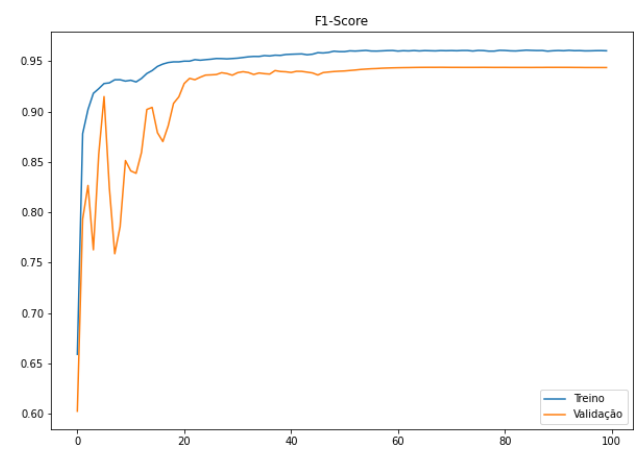

Figura 2. Aprendizado da arquitetura PSPNet com base da métrica F1-Score.

As Figuras 3 e 4 mostram resultados visuais do método proposto PSPNet. Podemos observar a imagem pré-processada, a segmentação do especialista e a segmentação do método proposto, respectivamente. A Figura 3 apresenta o melhor caso, e a Figura 4 apresenta o pior caso, baseado no valor de F1-Score obtido. A partir do melhor caso é possível notar que o resultado obtido com o modelo consegue obter um nível de detalhes melhor que o especialista, visto que as bordas são mais sutis e arredondadas que na segmentação do especialista.

\section{Conclusão}

Este trabalho apresenta um método automático para segmentação automática do músculo Longissimus dorsi a partir de imagens de ultrassom de pequenos ruminantes. Neste trabalho foram realizados experimentos em que diversos modelos de arquiteturas de CNNs foram testados e comparados. Quando comparadas, as CNNs apresentaram resultados semelhantes, no entanto, a arquitetura PSPNet se destacou das demais. 

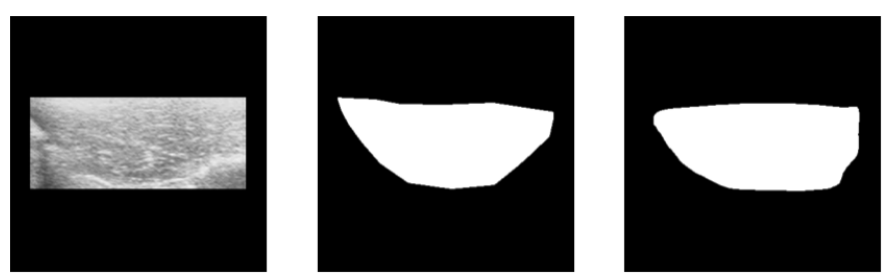

Figura 3. Resultado da segmentação do método proposto na imagem com maior valor de F1-Score (Da esquerda para direita, imagem pré-processada, a segmentação do especialista e a segmentação com o modelo proposto).
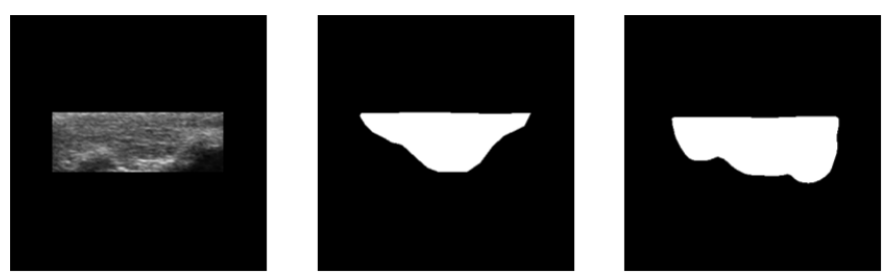

Figura 4. Resultado da segmentação do método proposto na imagem com menor valor de F1-Score (Da esquerda para direita, imagem pré-processada, a segmentação do especialista e a segmentação com o modelo proposto).

O presente estudo mostrou que a utilização de métodos automáticos para o reconhecimento de imagens ultrassonográficas é aceitável, pois, avaliação manual de imagens ultrassonográficas de pequenos ruminantes é uma atividade que pode ser longa, cansativa e trabalhosa. Essa metodologia pode trazer mais agilidade e precisão ao trabalho destes profissionais. Com base nos testes foi possível obter uma avaliação precisa da carcaça das imagens inseridas, o que poderá permitir ao produtor um diagnóstico correto das mensurações dos animais, com maior praticidade.

Como trabalhos futuros, iremos utilizar o resultado obtido na segmentação para realizar a predição dos valores de AOL e EGS. Para isso, serão extraídos atributos das imagens a partir da forma da músculo. Com isso, serão utilizados algoritmos de regressão e métricas como erro médio quadrático e coeficiente $\mathrm{R}^{2}$.

\section{Referências}

Araújo, R. L., de Araújo, F. H. D., and Silva, R. R. V. e. (2021). Automatic segmentation of melanoma skin cancer using transfer learning and fine-tuning. Multimedia Systems.

Cartaxo, F. Q. and Sousa, W. H. d. (2008). Correlações entre as características obtidas in vivo por ultra-som e as obtidas na carcaça de cordeiros terminados em confinamento. Revista Brasileira de Zootecnia, 37:1490-1495.

Chaurasia, A. and Culurciello, E. (2017). Linknet: Exploiting encoder representations for efficient semantic segmentation. In 2017 IEEE Visual Communications and Image Processing (VCIP), pages 1-4. IEEE.

Cutler, A., Cutler, D. R., and Stevens, J. R. (2012). Random forests. In Ensemble machine learning, pages 157-175. Springer. 
Daniel, H., González, G. V., García, M. V., Rivero, A. J. L., and De Paz, J. F. (2020). Non-invasive automatic beef carcass classification based on sensor network and image analysis. Future Generation Computer Systems, 113:318-328.

Font-i Furnols, M., Brun, A., Marti, S., Realini, C., Pérez-Juan, M., Gonzalez, J., and Devant, M. (2014). Composition and intramuscular fat estimation of holstein bull and steer rib sections by using one or more computed tomography cross-sectional images. Livestock Science, 170:210-218.

IBGE (2020). Pesquisa da pecuária municipal. Último acesso em 13 de outubro de 2020.

Jain, A. K. and Li, S. Z. (2011). Handbook of face recognition, volume 1. Springer.

Kempster, A., Cuthbertson, A., Harrington, G., et al. (1982). Carcase evaluation in livestock breeding, production and marketing. Granada Publishing Limited.

Lin, T.-Y., Dollár, P., Girshick, R., He, K., Hariharan, B., and Belongie, S. (2017). Feature pyramid networks for object detection. In Proceedings of the IEEE conference on computer vision and pattern recognition, pages 2117-2125.

Lindner, C., Wang, C.-W., Huang, C.-T., Li, C.-H., Chang, S.-W., and Cootes, T. F. (2016). Fully automatic system for accurate localisation and analysis of cephalometric landmarks in lateral cephalograms. Scientific reports, 6:33581.

Neto, A. D. B. (2010). Posicionamento estratégico do setor de carnes de caprinos e ovinos no mercado de carnes brasileiro. Revista Tecnologia \& Ciência Agropecuária, 4(4):81-85.

Nowozin, S. (2014). Optimal decisions from probabilistic models: the intersection-overunion case. In Proceedings of the IEEE conference on computer vision and pattern recognition, pages 548-555.

Oliver, A., Mendizabal, J., Ripoll, G., Albertí, P., and Purroy, A. (2010). Predicting meat yields and commercial meat cuts from carcasses of young bulls of spanish breeds by the seurop method and an image analysis system. Meat science, 84(4):628-633.

Peña, F., Santos, R., Juárez, M., Avilés, C., Domenech, V., González, A., Martínez, A., and Molina, A. (2014). The use of ultrasound scanning at different times of the finishing period in lean cattle. Livestock Science, 167:381-391.

Ronneberger, O., Fischer, P., and Brox, T. (2015). U-net: Convolutional networks for biomedical image segmentation. In International Conference on Medical image computing and computer-assisted intervention, pages 234-241. Springer.

ROSANOVA, C. (2004). Fatores favoráveis e limitantes ao desenvolvimento da cadeia produtiva da ovinocaprinocultura de corte no brasil. Monografia, Universidade Federal de Lavras.

Tauroco (2020). A utilização da técnica de ultrassonografia em tempo real para avaliação e seleção de características de carcaça em animais de corte.

Zhao, H., Shi, J., Qi, X., Wang, X., and Jia, J. (2017). Pyramid scene parsing network. In Proceedings of the IEEE conference on computer vision and pattern recognition, pages 2881-2890. 